\title{
Textbook of Voiding Dysfunction and Female Urology. 3rd ed.
}

\author{
By Korean Continence Society. Seoul: A-Plus Planning; 2015. 608 pp. KRW 100,000. ISBN: 979-11-951838-2-1
}

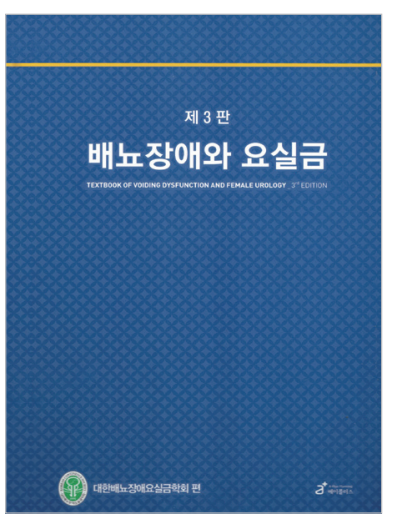

Voiding dysfunction and urinary incontinence have affected patients' quality of life over the years. Recently, however, increased attention to these areas has led to a better understanding of the anatomy, pathophysiology, and newer approaches to treatment. This textbook, the official textbook of the Korean Continence Society, provides information necessary for the understanding, diagnosis, and treatment of voiding dysfunction and female urology. In particular, the third edition has been reviewed and updated throughout, with emphasis on current references.

The book has four sections: understanding of the lower urinary tract, introduction of lower urinary tract dysfunction, lower urinary tract dysfunction and female urology, and research on lower urinary tract dysfunction. The latest concepts in anatomy, physiology, and pharmacology regarding the lower urinary tract are presented in the first two sections of the book.
The third section includes topics such as neurogenic bladder, overactive bladder, urinary incontinence, voiding dysfunction, benign prostatic hyperplasia, pelvic pain, pelvic organ prolapse, fistula, and female sexual dysfunction. In particular, research on lower urinary tract dysfunction is included as a separate section of the book, and there are two parts devoted to basic and clinical researches.

We all have much to learn in the area of voiding dysfunction and female urology. Although it is written in Korean, this book will help to build on the basic introduction and provide a framework towards better understanding of these diseases. The book will be invaluable for the trainee as well as the trained urologist who deal with lower urinary tract dysfunction on a daily basis. It will also be useful for nurse practitioners, medical students, and all other physicians who are interested in understanding voiding dysfunction and female urology.

Sung Tae Cho

Department of Urology, Hallym University College of Medicine E-mail: stcho@hallym.ac.kr 